\title{
AI_COVID: AUTOMATIC DIAGNOSIS OF COVID-19 USING FRONTAL CHEST X-RAY IMAGE
}

\author{
I. Allaouzi ${ }^{1}$, B. Benamrou ${ }^{2}$, A. Allaouzi ${ }^{3}$, M. Ouardouz ${ }^{2}$, and M. Ben Ahmed ${ }^{1}$ \\ 1LIST/Faculty of Sciences and Techniques, Abdelmalek Essaadi University, Tangier, Morocco \\ 2MMC/Faculty of Sciences and Techniques, Abdelmalek Essaadi University, Tangier, Morocco \\ 3LISAC/Faculty of Science Dhar El Mahraz, Sidi Mohamed Ben Abdellah University, Fez, Morocco
}

KEY WORDS: COVID-19, Chest X-ray, Artificial Intelligence, DenseNet-121, SVM, Transfer learning.

\begin{abstract}
:
With the continued growth of confirmed cases of COVID-19, a highly infectious disease caused by a newly discovered coronavirus called Severe Acute Respiratory Syndrome Coronavirus 2, or SARS-CoV-2, there is an urgent need to find ways to help clinicians fight the virus by reducing the workload and speeding up the diagnosis of COVID-19. In this work, we propose an artificial intelligence solution "AI_COVID" which can help radiologists to know if the lungs are infected with the virus in just a few seconds.
\end{abstract}

AI_COVID is based on a pre-trained DenseNet-121 model that detects subtle changes in the lungs and an SVM classifier that decides whether these changes are caused by COVID-19 or other diseases. AI_COVID is trained on thousands of frontal chest x-rays of people who have contracted COVID-19, healthy people, and people with viral or bacterial pneumonia. The experimental study is tested on 781 chest x-rays from two publicly available chest x-ray datasets COVID-19 radiography database and COVIDx Dataset. The performance results showed that our proposed model (DenseNet-121 + SVM) demonstrated high performance and yielded excellent results compared to the current methods in the literature, with a total accuracy of $99.74 \%$ and $98.85 \%$ for binary classification (COVID-19 vs. No COVID-19) and multi-class classification (COVID-19 vs. Normal vs. Pneumonia), respectively.

\section{INTRODUCTION}

COVID-19 is a highly infectious disease caused by a newly discovered coronavirus called Severe Acute Respiratory Syndrome Coronavirus 2, or SARS-CoV-2. The novel coronavirus appeared for the first time in Wuhan, China, in late December 2019, and quickly spread to all continents of the world. Accordingly, the World Health Organization (WHO) declared the ongoing outbreak as a global health emergency on 30 January 2020, and then a pandemic on 11 March 2020 (H. DS, 2019)

The SARS-CoV-2 virus is the third zoonotic coronavirus (i.e. it is transmitted between animals and people), after SARS-CoV and MERS-CoV, and the only one with pandemic potential. The virus settles in the human body during a period called incubation: this is the time from exposure to the development of symptoms of the disease. The incubation period is currently estimated to be between 2 and 14 days, most often around 5 days.

COVID-19 is transmitted from an infected person with SARSCoV-2 to another person mainly by direct contact through respiratory droplets expelled by the nose or mouth when a person coughs or sneezes, or by contact with contaminated objects and surfaces. Symptoms of COVID-19 are not specific because it affects different people in different ways. The disease presentation can range from no symptoms (asymptomatic) to respiratory symptoms including fever, dry cough, shortness of breath, and dyspnea. In more serious and critical cases, COVID19 can cause lasting damage to the lungs and other organs such as pneumonia, acute respiratory distress syndrome, kidney failure, and even death.

The rapid human-to-human transmission of COVID-19 constitutes the largest global health crisis of our time, with more than 219 million confirmed cases and over 4 million confirmed deaths globally as of September 7, 2021, with numbers continuing to rise. This situation puts countries on alert to do everything in their power to slow the spread of the virus by testing and treating patients, conducting contact tracing, limiting travel, quarantining citizens, and canceling large gatherings. These measures have reduced the workforce in all economic sectors and caused the loss of many jobs, which has sparked fears of an impending economic crisis and recession.

Faced with the widespread transmission of COVID-19, the deployment of the SARS-CoV-2 diagnostic test has varied considerably around the world, as it is contingent on the availability of testing supplies, laboratory capacity, and the time required to obtain results. Real-time reverse transcription polymerase chain reaction (RT-PCR) is the most common diagnostic test used to determine people currently infected by SARS-CoV-2. It works by detecting whether or not viral RNA is present in samples from individuals suspected of COVID-19. RT-PCR is highly specific but has a lower sensitivity of $65-95 \%$ (Bai., H. X., Hsieh., B., Xiong., Z., 2020). This means that the test can be negative even when the patient is infected. Another problem is that the RT-PCR test is an expensive and timeconsuming process that takes up to two days between taking a sample to a specialized laboratory and getting a diagnosis. To overcome these limitations, radiologists have raced to find out how medical imaging can help to screen or speed up the diagnosis of infected people. Indeed, frontline health workers have found the diagnostic value of imaging to be increasingly valuable, resulting in clinical diagnosis and monitoring of COVID-19. The primary findings of COVID-19 on chest X-ray (CXR) and computed tomography (CT) include lung consolidation and ground-glass opacities. Despite CXR and CT chest features being common in COVID-19 patients, CT imaging is highly recommended as it is very sensitive to early detection of disease, assessment of the nature and extent of lesions, and discovery of subtle changes that are often not visible on CXR (Yang., W., 2020). However, due to infection 
control issues associated with transporting patients to CT suites, the inefficiencies introduced in CT room decontamination, and the lack of availability of CT scans in some parts of the world, CXR is still useful because it is relatively inexpensive, exposing patients to less radiation, and is one of the most widely available and accessible $\mathrm{x}$-ray exams, as it is considered standard equipment in most healthcare systems. Most importantly, the presence of portable CXR systems that allow imaging directly in an isolation room, without the need to transport patients with suspected or confirmed COVID-19 to a room with fixed imaging systems, thereby limiting the contamination of radiology equipment and environment.

Unlike the exponential increase in the number of infected patients, especially in the most impacted areas, there is a severe shortage of expert radiologists to interpret COVID-19 CXRs, as visual indicators can be subtle. In this regard, artificial intelligence (AI) systems based on deep learning have been developed to mitigate the radiologist's workload and accelerate the COVID-19 detection process by interpreting the CXR images quickly and accurately.

\section{DEEP LEARNING FOR COVID-19 DETECTION USING CXR IMAGES}

Deep learning techniques have proven to be very effective in various medical imaging tasks by providing accurate and efficient diagnostics. The Convolutional Neural Network $(\mathrm{CNN})$ is the most powerful and commonly applied technique in detecting and classifying radiological abnormalities on CXRs. Inspired by the recent success of $\mathrm{CNN}$ in thorax disease diagnosis, including pneumonia (Guan., Q., 2018) (Rubin., J., 2018) (Allaouzi., I. and Ben Ahmed., M., 2019), various CNNbased works have been proposed to contribute to the large-scale screening of patients infected with COVID-19 using CXRs:

In (Wang., L., 2020), the authors introduced a deep CNN called COVID-Net, which achieved an accuracy of $93.3 \%$ on a test dataset containing 100 CXR images with COVID-19 pneumonia, $100 \mathrm{CXRs}$ with pneumonia, and $100 \mathrm{CXRs}$ with non-COVID-19 pneumonia. In addition, the authors released the dataset used to train and evaluate the proposed COVID-Net to aid fight COVID-19. In (Apostolopoulos., I., D., 2020), an overall accuracy of $97.82 \%$ in the detection of COVID-19 is achieved utilizing transfer learning with five CNNs. The training and evaluation procedure was performed with 10 -foldcross validation on a data collection of 224 CXRs with confirmed COVID-19, 700 CXRs with confirmed common pneumonia, and 504 CXRs of normal conditions. In (Narin., A., 2020), three different pre-trained CNN based models (ResNet50, InceptionV3, and Inception-ResNetV2) have been proposed. These models were trained and tested using a very small dataset of 50 normal CXRs and 50 COVID-19 CXRs. The performance results showed that the pre-trained ResNet50 model gave the highest accuracy of $98 \%$. Likewise, in (Chowdhury., M. E. H., 2020), four different popular pretrained CNN models were trained and tested on a database containing a mixture of 190 COVID-19, 1345 viral pneumonia, and 1341 normal CXR images. The performance results showed that the SqueezeNet model yielded the highest accuracy of $98 \%$ for binary classification (normal vs COVID-19) and multi-class classification (COVID-19 vs. normal vs. pneumonia), using a test dataset with 60 images of each category. The authors have also prepared a relatively large database of X-ray images of normal, viral pneumonia, and COVID-19 positive pneumonia and have made it publicly available so that other researchers can benefit from it. While in (Sethy., P. K., and Behera, S. K., 2020), the authors used a pre-trained ResNet50 model with a support vector machine (SVM) classifier to classify CXR images into the following three categories COVID-19, pneumonia, and normal. The proposed model was trained and tested on a dataset of 1809 CXRs, and achieved an overall test accuracy of $98 \%$ using 127 images from each category. In (Ozturk., T., 2020), the DarkNet model was used as a classifier for binary classification (COVID-19 vs. No-Findings) and multi-class classification (COVID-19 vs. No-Findings vs. Pneumonia). The proposed DarkNet model was developed using a total of 1125 images (125 COVID-19, 500 pneumonia, and 500 no-findings) and achieved an accuracy of $98.08 \%$ and $87.02 \%$ for binary and three classes, respectively.

\section{MATERIAL AND METHODS}

\subsection{CXR Dataset:}

The performance of deep learning models for image classification is limited by the availability of large-scale image datasets. However, obtaining large datasets of COVID-19 chest $\mathrm{X}$-ray images remains a challenge.

There are few publicly available COVID-19 CXR datasets that are sufficiently large:

3.1.1 COVID-19 Radiography Database: A team of researchers from Qatar University, Doha, Qatar and the University of Dhaka, Bangladesh along with their collaborators from Pakistan and Malaysia in collaboration with medical doctors have created a database of chest X-ray images for COVID-19 positive cases along with normal and viral pneumonia images (Chowdhury., M. E. H., 2020). The COVID-19 CXR images were collected from the Italian society of medical and interventional radiology (SIRM) COVID-19 database, Novel Corona Virus 2019 Dataset (Cohen., J. P., 2020), and images extracted from 43 different publications. Whereas normal and viral pneumonia images were adopted from the Chest X-Ray Images (pneumonia) database (Mooney., P., 2018).

The COVID-19 radiography database contains 1024x1024 Portable Network Graphics (PNG) Images of 219 COVID-19 positive CXR, 1341 normal CXR, and 1345 viral pneumonia CXR.

3.1.2 COVIDx Dataset: The current COVIDx dataset includes 13975 CXR images of 13870 patients collected from the following publically available datasets:

- COVID-19 Image Data Collection (Cohen., J.P., 2020).

- Figure 1 COVID19 Chest X-ray Dataset Initiative (Chung, 2020).

- RSNA Pneumonia Detection Challenge dataset, which used publicly available CXR data from (Wang., X., 2017).

- COVID-19 radiography database (Chowdhury., M.E. H., 2020).

- Actualmed COVID-19 Chest X-ray Dataset (Chung., A., 2020).

The database contains chest $\mathrm{x}$-ray images of various sizes and formats including 8066 normal CXR images, 5551 pneumonia CXR images, and 358 COVID-19 CXR images. 

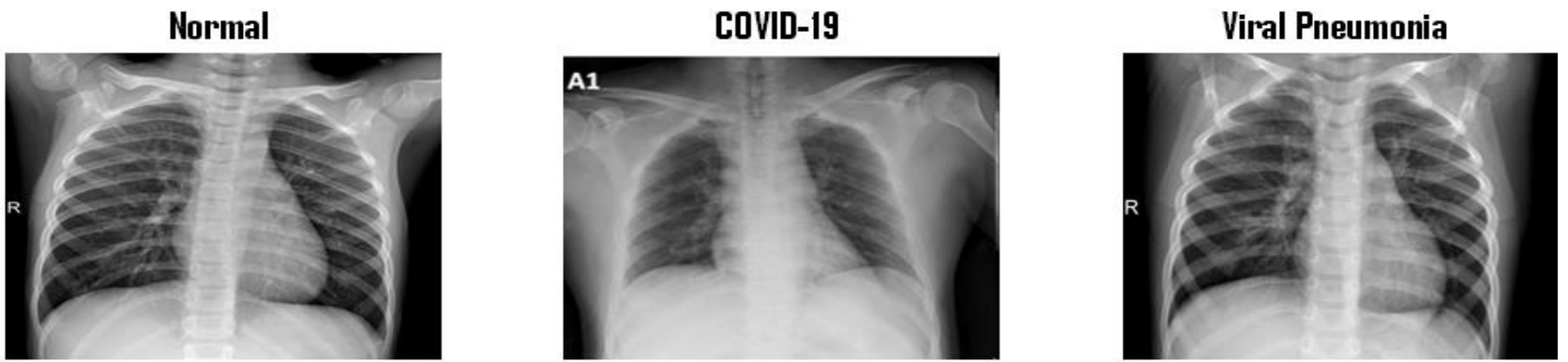

Figure 1. Illustrative examples of Chest X-ray samples from COVID-19 radiography database.

\subsection{The Proposed Model}

The idea behind our approach is to combine the strength of pretrained CNN in extracting relevant features from images and the power of the traditional machine learning classifier in the binary classification task. Our motivation comes from the very significant results we have obtained using such an approach to detect the presence of 14 common chest diseases from CXR images (Allaouzi., I. and Ben Ahmed., M., 2019).

Following the aforementioned approach, the proposed model consists of the DenseNet-121 network pre-trained on CHESTXRAY14 (J. Rubin, 2018), a large dataset of frontal CXR images, and one of the most powerful binary classifier, the support vector machine (SVM). As illustrated in Figure 2, the model takes as input a CXR image which first undergoes certain preprocessing techniques, such as resizing the CXR image to the format required by DenseNet-121 (224x224 pixels), and then the CXR image is passed to DenseNet-121 network which is used as a fixed feature extractor by removing its last fullyconnected layer to get as output a 1024-dimensional feature vector. Finally, the extracted features are fed to the SVM classifier in order to provide accurate diagnostics for binary classification (COVID-19 vs. no COVID-19) and multi-class classification (normal vs. COVID-19 vs. pneumonia).

Figure 2. The proposed model pipeline.

\subsection{Classification Performance}

The evaluation method is a key factor in assessing classification performance and guiding classifier modeling. It is based on the counts of test records correctly and incorrectly predicted by the model.

Different metrics can be used to evaluate the prediction of the classification model, however, the most commonly used in the literature are:

3.3.1 Confusion Matrix: The confusion matrix is a good option for reporting the results of M-class classification problems because it allows a clear tabular representation of the relationship between predicted and actual values, and what type of errors are being made. Figure 3 shows the confusion matrix in a 2-class classification problem.

The green diagonal indicates correct predictions and the pink diagonal represents the incorrect predictions. As depicted, the confusion matrix is made up of:

- True positive (TP): The sample is positive and it is classified as positive.

- False negative (FN): The sample is positive and it is classified as negative.

- True negative (TN): The sample is negative and it is classified as negative.

- False positive (FP): The sample is positive and it is classified as positive.

\section{True/Actual Class}

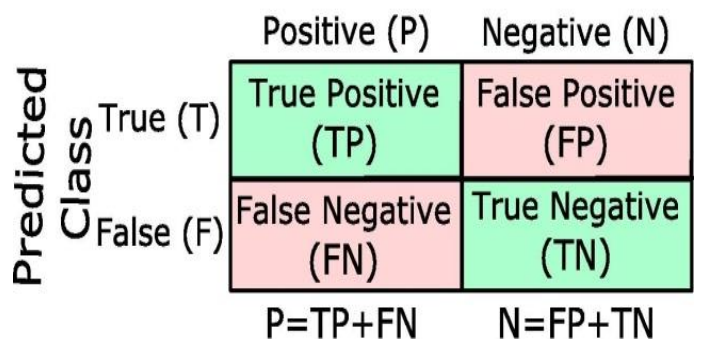

Figure 3. Confusion matrix in a 2-class classification. 


\subsubsection{Accuracy:}

Accuracy is one of the criteria for evaluating classification models. Accuracy refers to the ratio of the number of correct predictions made by the classifier to the total number of input samples. Formally, the accuracy is defined as:

$$
\text { Accuracy }=\frac{\mathrm{TP}+\mathrm{TN}}{\mathrm{TP}+\mathrm{FP}+\mathrm{TN}+\mathrm{FN}}
$$

\subsubsection{Sensitivity and Specificity:}

Sensitivity and specificity are two important concepts in medical testing where even a small false positive or false negative can have a serious effect. Sensitivity measures the ability of a test to correctly identify people with the condition that's being tested for (also known as the "true positive rate"), whereas specificity measures the ability of the test to correctly identify those without the condition that's being tested for (also known as the "true negative rate"). These measures are defined as:

$$
\begin{aligned}
& \text { Sensitivity }=\frac{\mathrm{TP}}{\mathrm{TP}+\mathrm{FP}} \\
& \text { Specificity }=\frac{\mathrm{TN}}{\mathrm{TN}+\mathrm{FN}}
\end{aligned}
$$

\section{EXPERIMENT AND RESULTS:}

In our experiment, we address two types of classification tasks: binary classification and multi-class classification. More precisely, the binary classification task included COVID-19 and no COVID-19 infection, while the multi-class classification task included three classes: normal, COVID-19, viral and bacterial

\begin{tabular}{|c|c|c|c|c|}
\hline \multirow{4}{*}{ Source } & \multicolumn{4}{|l|}{ CXR Images } \\
\hline & \multirow{3}{*}{ COVID-19 } & \multicolumn{3}{|c|}{ No COVID-19 } \\
\hline & & \multicolumn{2}{|c|}{ Pneumonia } & \multirow{2}{*}{ Normal } \\
\hline & & Viral & Bacterial & \\
\hline COVID-19 radiography database & 219 & 1345 & - & 1342 \\
\hline COVID-19 radiography database and COVIDx dataset & 297 & 1345 & 2330 & 1342 \\
\hline
\end{tabular}
pneumonia. The distribution of CXR images amongst the different infection types is shown in Table 1.

Table 1. The distribution of CXR images amongst the different infection types.

For our experiment, we randomly split the dataset into training (4732 images), and test (781 images). The dataset consists of 5513 CXR images from the COVID-19 radiography database and COVIDx dataset. Table 2 illustrates the distribution of CXR images in the training and test sets.

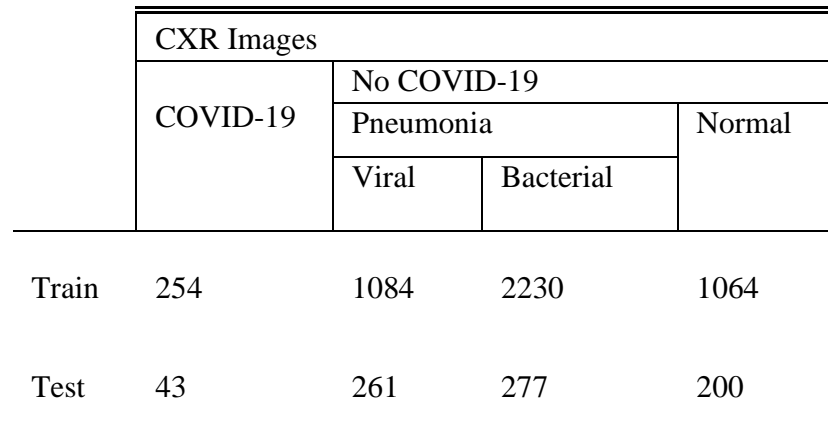

Table 2. The distribution of CXR images in training and test sets.

We trained the DenseNet-121 model on our dataset, using initial weights obtained from the pre-trained network, on CHESTXRAY14, which gives a good starting point compared to the randomly initialized weights. We used a mini-batch size of 8 samples, a number of epochs equal to 10 , the categorical crossentropy as a loss function, and the Adam optimizer with an initial learning rate of 0.001 . As this trained model is used only as a feature extractor, we peeled off the last fully connected layer (4D fully connected, Softmax) and used the output from all the previous layers (a fixed feature vector of 1024D) as features for an SVM classifier with RBF kernel.

\subsection{Binary Classification Task: (COVID-19 vs. No COVID- 19)}

For the binary classification task, the SVM classifier is trained on 254 COVID-19 and 4478 No COVID-19 CXR images, and it is tested on 43 COVID-19 and 738 No COVID-19 CXR images. The confusion matrix of the classification results is illustrated in Figure 4. In addition, the classification performance in terms of accuracy, sensitivity, and specificity is given in Table 3.

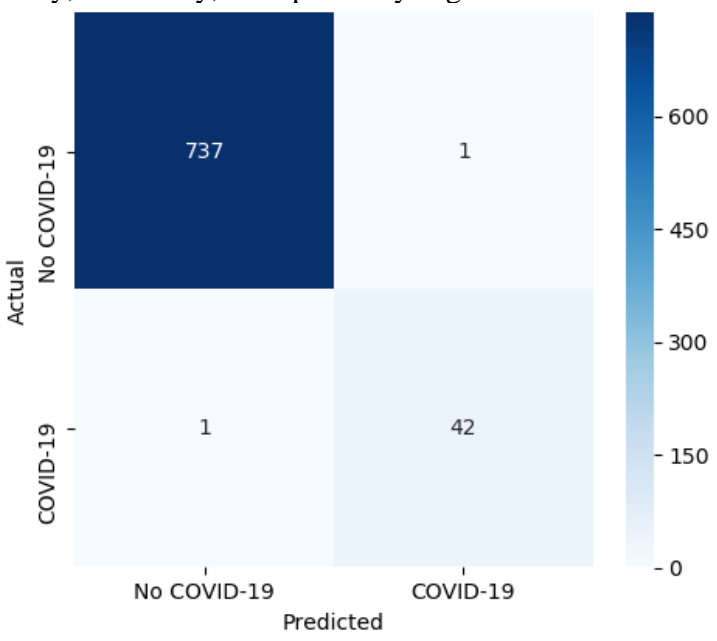

Figure 4. Confusion matrix of the binary classification. 


\begin{tabular}{l|lcl}
\hline \hline \multirow{2}{*}{ Class } & \multicolumn{3}{l}{ Evaluation metrics } \\
\cline { 2 - 4 } & $\begin{array}{l}\text { Overall } \\
\text { Accuracy }\end{array}$ & Sensitivity & Specificity \\
\hline COVID-19 & $99.74 \%$ & $97.67 \%$ & $99.86 \%$ \\
No COVID-19 & & $99.86 \%$ & $97.67 \%$ \\
\hline \hline
\end{tabular}

Table 3. The performance results of the binary classification (COVID-19 vs. No COVID-19).

4.2 Multi-class Classification Task: (COVID-19 vs. Pneumonia vs. Normal)

For the multi-class classification task, the SVM classifier is trained on 254 COVID-19, 3314 Pneumonia, and 1064 Normal CXR images, and it is tested on 43 COVID-19, 538 Pneumonia, and 200 Normal CXR images. The confusion matrix of the classification results is illustrated in Figure 5. In addition, the classification performance in terms of accuracy, sensitivity, and specificity is given in Table 4 .

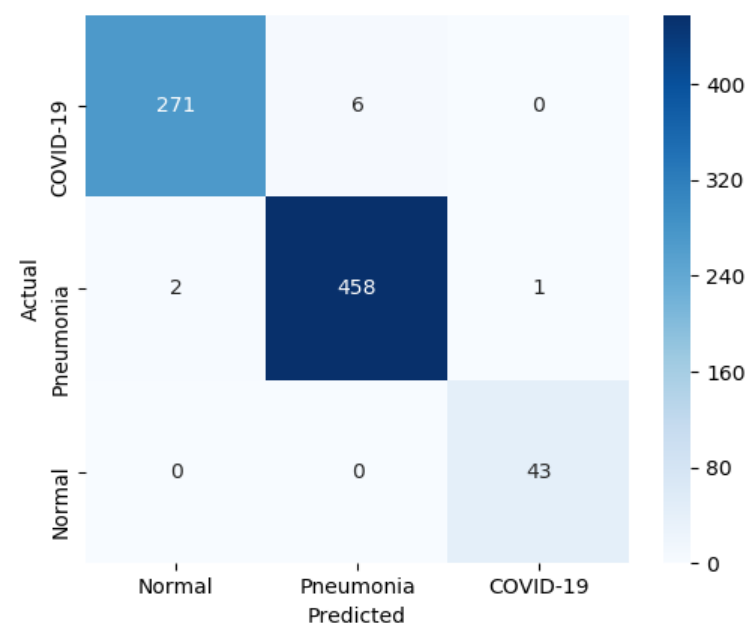

Figure 5. Matrix of the 3-class classification.

\begin{tabular}{l|lll}
\hline \hline \multirow{2}{*}{ Class } & \multicolumn{3}{|l}{ Evaluation metrics } \\
& $\begin{array}{l}\text { Overall } \\
\text { Accuracy }\end{array}$ & Sensitivity & $\begin{array}{l}\text { Specificit } \\
\text { y }\end{array}$ \\
\hline COVID-19 & & $97.73 \%$ & $100 \%$ \\
Pneumonia & $98.85 \%$ & $98.71 \%$ & $99.06 \%$ \\
Normal & & $99.27 \%$ & $98.83 \%$ \\
\hline \hline
\end{tabular}

Table 4. The performance results of the three-class classification (COVID-19 vs. Normal vs. Pneumonia).

The performance results showed that our proposed model (DenseNet-121 + SVM) demonstrated high performance and gave excellent results with an overall accuracy of $99.74 \%$ and 98.85\% for binary classification (COVID-19 vs. No COVID19) and multi-class classification (COVID-19 vs. Normal vs. Pneumonia), respectively, using a test dataset with 781 images. As shown in Tables 3 and 4, the COVID-19 sensitivity and specificity values for binary and 3-class classification are $97.67 \%, 99.86 \%, 97.73 \%$, and $100 \%$, respectively.
More importantly, the results obtained by our proposed method were compared with the results of the state of the art using the following table. After analyzing Table 5, we found that we obtained the highest performance compared to studies in the literature for both binary classification and 3-class classification.

\begin{tabular}{|c|c|c|c|}
\hline Method & Number of cases & Task & $\begin{array}{l}\text { Overall } \\
\text { accuracy }\end{array}$ \\
\hline $\begin{array}{l}\text { COVID- } \\
\text { Net (Wang., } \\
\text { L., 2020) }\end{array}$ & $\begin{array}{l}\text { 100COVID-19(+) } \\
\text { 100Pneumonia } \\
100 \text { Normal }\end{array}$ & $\begin{array}{l}\text { 3-class } \\
\text { classification }\end{array}$ & $93.3 \%$ \\
\hline $\begin{array}{l}\text { VGG-19 } \\
\text { (Apostolop } \\
\text { oulos., I. I. } \\
\text { D., 2020) }\end{array}$ & $\begin{array}{l}224 \text { COVID-19(+) } \\
700 \text { Pneumonia } \\
504 \text { Normal } \\
224 \text { COVID-19(+) } \\
1204 \text { COVID-19(-) }\end{array}$ & $\begin{array}{l}\text { 3-class } \\
\text { classification } \\
\text { Binary } \\
\text { classification }\end{array}$ & $98.75 \%$ \\
\hline $\begin{array}{l}\text { ResNet- } \\
\text { 50(Narin., } \\
\text { A., 2020) }\end{array}$ & $\begin{array}{l}\text { 50COVID-19(+) } \\
50 \text { COVID-19 }(-)\end{array}$ & $\begin{array}{l}\text { Binary } \\
\text { classification }\end{array}$ & $98 \%$ \\
\hline $\begin{array}{l}\text { SqueezeNet } \\
\text { (Chowdhur } \\
\text { y, M. E. } \\
\text { H., 2020) }\end{array}$ & $\begin{array}{l}60 \text { COVID-19(+) } \\
60 \text { Pneumonia } \\
60 \text { Normal } \\
60 \text { COVID-19(+) } \\
60 \text { COVID-19 }(-)\end{array}$ & $\begin{array}{l}\text { 3-class } \\
\text { classification } \\
\text { Binary } \\
\text { classification }\end{array}$ & $95.19 \%$ \\
\hline $\begin{array}{l}\text { ResNet- } \\
\text { 50+SVM } \\
\text { (Sethy., P. } \\
\text { K., 2020) }\end{array}$ & $\begin{array}{l}\text { 127COVID-19(+) } \\
\text { 127Pneumonia } \\
127 \text { Normal }\end{array}$ & $\begin{array}{l}\text { 3-class } \\
\text { classification }\end{array}$ & $98 \%$ \\
\hline $\begin{array}{l}\text { DarkNet } \\
\text { (Ozturk.,T., } \\
\text { 2020) }\end{array}$ & $\begin{array}{l}\text { 125COVID-19(+) } \\
500 \text { Pneumonia } \\
500 \text { Normal } \\
125 \text { COVID-19(+) } \\
500 \text { No-Findings }\end{array}$ & $\begin{array}{l}\text { 3-class } \\
\text { classification } \\
\text { Binary } \\
\text { classification }\end{array}$ & $87.02 \%$ \\
\hline $\begin{array}{l}\text { Our } \\
\text { proposed } \\
\text { method: } \\
\text { DenseNet- } \\
\text { 121+SVM }\end{array}$ & $\begin{array}{l}\text { 43COVID-19(+) } \\
538 \text { Pneumonia } \\
200 \text { Normal } \\
43 \text { COVID-19(+) } \\
738 \text { COVID-19 }(-)\end{array}$ & $\begin{array}{l}\text { 3-class } \\
\text { classification } \\
\text { Binary } \\
\text { classification }\end{array}$ & $98.85 \%$ \\
\hline
\end{tabular}

Table 5. Comparison to the state-of-the-art methods.

\section{CONCLUSION}

Faced with the continuous growth of confirmed COVID-19 cases, there is an urgent need to find ways to help clinicians fight the virus by reducing the workload and speeding up the diagnosis of COVID-19. In this regard, we offer an artificial intelligence solution "AI_COVID" that can help radiologists know if the lungs are infected with the virus in just a few seconds.

The solution is able to identify COVID-19 cases from frontal chest radiographic images. It is based on a combination of a pretrained DenseNet-121 model that detects subtle changes in the lungs and a support vector machine classifier which decides whether these changes are caused by COVID-19 or other diseases. Our proposed solution showed promising performance outcomes and outperformed current state-of-the-art methods by achieving an overall accuracy of $99.74 \%$ and $98.85 \%$ for binary classification (COVID-19 vs. No COVID-19) and multi-class classification (COVID-19 vs. Normal vs. Pneumonia), respectively. 
This work requires the collaboration of health professionals mainly radiologists, for this, we made our solution available online in order to be assessed and validated by radiologists (see, Figure 6).

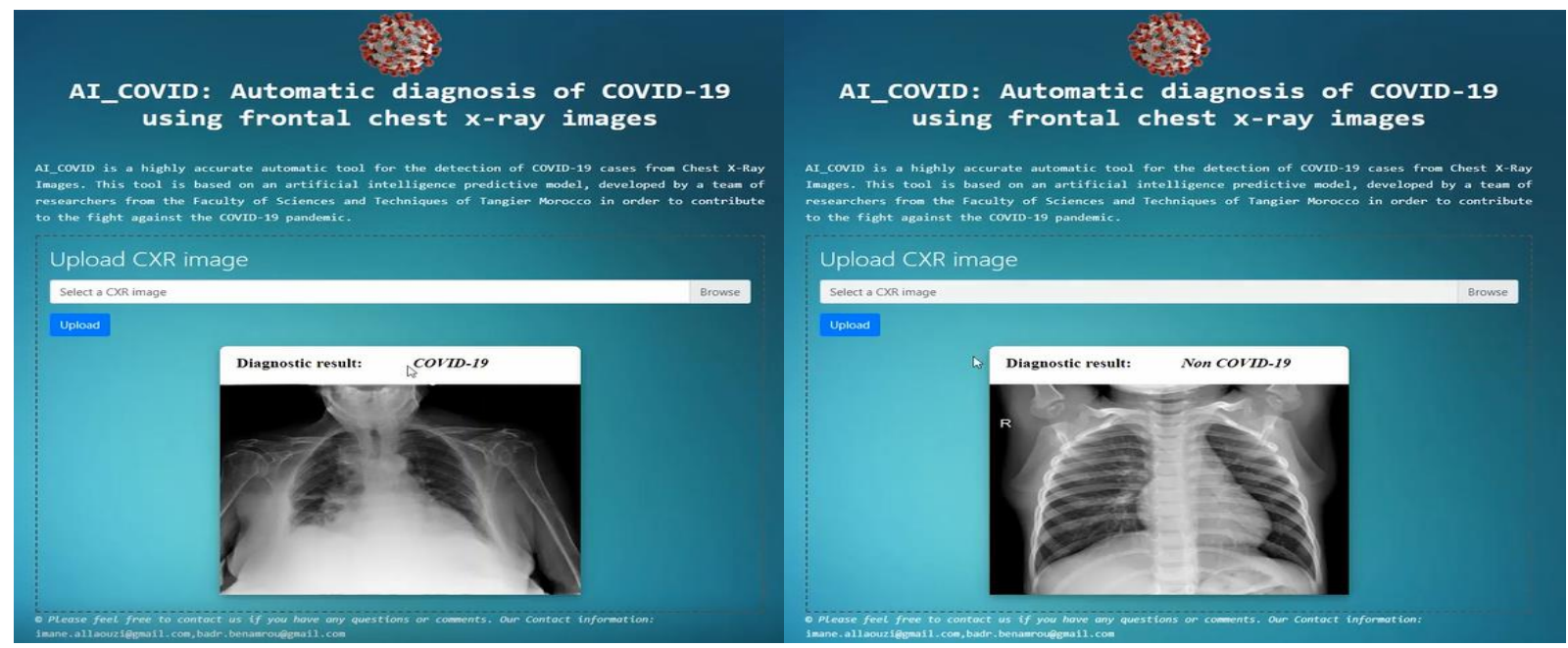

Figure 6. Example of two x-ray images diagnosed by the AI COVID solution.

\section{REFERENCES}

DS, H., EI, A., TA, M., 2020. The continuing 2019-nCoV epidemic threat of novel coronaviruses to global health-The latest 2019 novel coronavirus outbreak in Wuhan, China. Infect Dis Journal (91)1, 264-266.

WHO Director-General's opening remarks at the media briefing on COVID-19, World Health Organization (WHO) (Press release). 2020 Mar 11. Retrieved 2020 March 12. [Online]. Available: $\quad$ https://www.who.int/dg/speeches/detail/whodirector-general-s-opening-remarks-at-the-media-briefing-oncovid19---27-march-2020

Novel Coronavirus (2019-nCoV) Situation Report-7, World Health Organization (WHO), Jan. 2020.

China's National Health Commission news conference on coronavirus, Al Jazeera, Jan. 2020.

2019 Novel Coronavirus (2019-nCoV) Situation Summary, Centers for disease control and prevention, 2020 Jan 3. [Online]. Available: https://www.cdc.gov/coronavirus/2019ncov/ index.html

Bai., H. X., Hsieh., B., Xiong., Z., 2020. Performance of radiologists in differentiating COVID-19 from viral pneumonia on chest CT. Radiology (296)2, E46-E54, doi: 10.1148/radiol.2020200823

Yang., W., Sirajuddin., A., Zhang., X., 2020. The role of imaging in 2019 novel coronavirus pneumonia (COVID-19), Eur Radiol (30)9, 4874-4882, doi: 10.1007/s00330-020-068274

Guan., Q., Huang., Y., Zhong., Z., Zheng., Z., Zheng., L., Yang., Y., 2018. Diagnose like a radiologist: Attention guided convolutional neural network for thorax disease classification, arXiv preprint arXiv:1801.09927.
Rubin., J., Sanghavi., D., Zhao., C., Lee., K., Qadir., A., XuWilson., M., 2018. Large Scale Automated Reading of Frontal and Lateral Chest X-Rays using Dual Convolutional Neural Networks, arXiv preprint arXiv:1804.07839.

Allaouzi., I., Ben Ahmed., M., 2019. A Novel Approach for Multi-Label Chest X-Ray Classification of Common Thorax Diseases, IEEE Access (7)1, 64279-64288, doi: 10.1109/ACCESS.2019.2916849.

Wang., L., Lin., Z. Q., Wong., A., 2020. COVID-Net: A Tailored Deep Convolutional Neural Network Design for Detection of COVID-19 Cases from X-ray Images, Sci Rep (10) 19549, doi: 10.1038/s41598-020-76550-Z

Apostolopoulos., I. D., Mpesiana., T.A., 2020. COVID-19: Automatic Detection from X-Ray Images Utilizing Transfer Learning with Convolutional Neural Networks. Phys Eng Sci Med, (43)1, 635-640, doi: 10.1007/s13246-020-00865-4.

Narin., A., Kaya., C., Pamuk., Z., 2020. Automatic Detection of Coronavirus Disease (COVID-19) Using X-Ray Images and Deep Convolutional Neural Networks, arXiv preprint arXiv:2003.10849.

Chowdhury, M. E. H., 2020. Can AI Help in Screening Viral and COVID-19 Pneumonia?. IEEE Access (8), 132665-132676, doi: 10.1109/ACCESS.2020.3010287.

Sethy., P. K., Behera., S. K., 2020. Detection of Coronavirus Disease (COVID-19) Based on Deep Features. Preprints 2020 , 2020030300, doi: 10.20944/preprints202003.0300.v1.

Ozturk., T., Talo., M., Yildirim., E. A., Baloglu., U. B., Yildirim , O., Acharya., U. R., 2020. Automated detection of COVID-19 cases using deep neural networks with X-ray images, Computers in Biology and Medicine (121) 103792, Jun. 2020, doi: 10.1016/j.compbiomed.2020.103792

SIRM COVID-19 Database, Apr, 2020. [Online]. Available: https://www.sirm.org/category/senza-categoria/covid-19/ 
Cohen., J. P., Morrison., P., Dao., L., 2020. COVID-19 image data collection, [Online] Available: https://github.com/ieee8023/covid-chestxray-dataset.

Mooney., P., 2018. Chest X-Ray Images (Pneumonia), [Online] Available: https://www.kaggle.com/paultimothymooney/chestxray-pneumonia.

Chung, 2020. Figure 1 COVID-19 chest x-ray data initiative. [Online] Available: https://github.com/agchung/Figure1COVID-chestxraydataset.

Radiological Society of North America, 2019, RSNA pneumonia detection challenge. [Online]. Available: https://www.kaggle.com/c/rsnapneumonia-detection challenge/data.
Wang., X., Peng., Y., Lu., L., Lu., Z., Bagheri., M., Summers., R., 2017. Chestx-ray8: Hospital-scale chest X-ray database and benchmarks on weakly-supervised classification and localization of common thorax diseases. IEEE Conference on Computer Vision and Pattern Recognition (CVPR), 3462-3471. Chung., A., 2020. Actual med COVID-19 chest x-ray data initiative. [Online]. Available: https://github.com/agchung/Actualmed-COVID-chestxraydataset. 